\title{
Assessment of In Vivo Clinical Product Performance of a Weak Basic Drug by Integration of In Vitro Dissolution Tests and Physiologically Based Absorption Modeling
}

\author{
Xuan Ding, ${ }^{1}$ Ivelina Gueorguieva, ${ }^{1}$ James A. Wesley, ${ }^{1}$ Lee J. Burns, ${ }^{1}$ and Carrie A. Coutant ${ }^{1,2}$
}

Received 16 March 2015; accepted 8 June 2015; published online 1 July 2015

\begin{abstract}
Effective integration of in vitro tests and absorption modeling can greatly improve our capability in understanding, comparing, and predicting in vivo performances of clinical drug products. In this case, we used a proprietary drug candidate galunisertib to describe the procedures of designing key in vitro tests, analyzing relevant experimental and trial data, and integrating them into physiologically based absorption models to evaluate the performances of its clinical products. By simulating the preclinical study result, we estimated high in vivo permeability for the drug. Given the high sensitivity of its solubility to $\mathrm{pH}$, supersaturation may play an important role in the absorption of galunisertib. Using the dynamic dissolution test, i.e., artificial stomach-duodenum (ASD) model and simulation, we concluded galunisertib in solution or tablet products could maintain supersaturation during the transit in the gastrointestinal tract (GIT). A physiologically based absorption model was established by incorporating these key inputs in the simulation of Trial 1 results of galunisertib solution. To predict the performance of three tablet products, we developed z-factor dissolution models from the multi-pH USP dissolution results and integrate them into the absorption model. The resultant biopharmaceutical models provided good prediction of the extent of absorption of all three products, but underestimated the rate of absorption of one tablet product. Leveraging the ASD result and optimization with the dissolution model, we identified the limitation of the model due to complexity of estimating the dissolution parameter $z$ and its in vitro-in vivo correlation.
\end{abstract}

KEY WORDS: absorption; dissolution; physiologically based model; z-factor.

\section{INTRODUCTION}

Oral absorption of solid dosage forms is a dynamic complex process affected by physicochemical properties related to the compound, formulation factors, and their interaction with gastrointestinal (GI) physiology (1). Dissolution or release from formulations, gastric emptying and intestinal transit of drug solute and/or solid particles, and transport of solute across the gut wall represent the most important and mechanistically distinct processes taking place during drug absorption. Understanding these processes in general and for a specific drug product is a prerequisite for identifying the critical success factors for pharmaceutical product development, and forming proper strategies for product design and optimization. Transport of drug solute occurs primarily in the small intestine, which includes passive diffusion through transcellular and paracellular pathways, and active transport processes mediated by various carriers (2-4). The passive diffusion is governed by permeability of the drug, surface area of the intestine available for drug absorption,

\footnotetext{
${ }^{1}$ Lilly Research Laboratory, Lilly Corporate Center, Eli Lilly and Company, Indianapolis, Indiana 46285, USA.

${ }^{2}$ To whom correspondence should be addressed. (e-mail: cacoutant@lilly.com)
}

and drug concentration gradient across the absorptive cell membrane (2). Permeability determines the rate at which dissolved drug can enter the enterocytes during the finite time of intestinal transit. For drug solids that require dissolution preceding the diffusion, high permeability enables an efficient sink condition to accelerate the dissolution. Biological membranes are generally more permeable to hydrophobic molecules. Optimization of permeability is usually performed during drug discovery stage through structural modification or prodrugs to increase the hydrophobicity (5). Given the heterogeneity in terms of cellular structures and functions, constant changes such as $\mathrm{pH}$ and secretion, and complex movement manifested by phased gastric motility, peristalsis, and segmental contraction in the intestine, drug barely goes on an easily predictable transit in the gastrointestinal tract (GIT) (6).

According to the Noyes-Whitney equation, the dissolution rate of a solid drug particle is determined by its surface area, drug solubility in the media at sink condition, diffusion coefficient, and diffusion layer thickness (7). For a slowly dissolving drug, micronization, ionization, or solubilization through formulation approaches could effectively resolve the issues that are caused by insufficient surface area or solubility (8-10). Hence, dissolution has long been recognized for its importance in evaluating and comparing in vivo performances of solid dosage forms (11). The ability 
of in vitro dissolution tests to predict the in vivo performances is primarily through the correlation between in vitro dissolution results and a relevant in vivo response, e.g., absorption, bioavailability, or the plasma concentration profile (12). For this utility, biorelevant dissolution tests are often recommended for their deliberate simulation of the in vivo environment where the drug is released from the formulation. Such a system often accounts for $\mathrm{pH}$ conditions, key components, and volume of the GI contents, transit times, and motility pattern to reflect the physiological conditions in the $\operatorname{GIT}(13,14)$. Dynamic dissolution systems aim to improve the ability of replicating the dynamic aspects of in vivo dissolution that are associated with the transit of dosage forms through a rapidly changing GI luminal environment (15). For instance, multicompartment dissolution models, represented by artificial stomach-duodenum model (ASD), find some use in evaluating the effect of gastric emptying on drug dissolution, and the propensity for supersaturation-precipitation of weak bases upon transfer from an acidic gastric compartment to a separate duodenal compartment. Supersaturation could enhance the apparent solubility of the basic drug in the intestine when it moves from a more soluble environment with lower $\mathrm{pH}$ (i.e., typical fasted stomach) to a less favorable environment with higher $\mathrm{pH}$ (i.e., intestine) (16-18). Coupled with high permeability for a Biopharmaceutical Classification System (BCS) II drug, supersaturation may greatly reduce the limit of solubility on its absorption (19).

The development of computational oral absorption modeling has significantly advanced predictive tools for oral drug product in vivo performance (20-22). By means of integrating the physicochemical properties of the drug obtained experimentally, system physiology information, and formulation characteristics, absorption modeling is able to delineate the complex mechanisms underlying drug absorption, and hence acquires the power of accurately predicting the oral drug product performance, and identifying the key parameters for potential performance improvement. More and more case examples have been reported to demonstrate various applications of such in silico-in vitro-in vivo approaches in modern pharmaceutical drug product development under the principle of Quality by Design $(23,24)$. For successful application of such an approach, robust and useful models are built on quality inputs and valid assumptions, which can be achieved through well-designed experiments and/or studies, and comprehensive analyses of these experimental and study results. We hereby used a proprietary drug candidate to describe the procedures of diligently analyzing relevant experimental data and integrating them into physiologically based absorption models to predict the performances of three immediate-release tablets. Subsequently, we critically assessed the predictability of these models against the clinical results. Using the physiologically based models, we shed light on how the prediction of in vivo performance could be impacted by the uncertainty of key model inputs such as dissolution parameters.

\section{EXPERIMENTAL SECTION}

\section{Materials}

Galunisertib (as shown in Fig. 1) was discovered and is under development by Eli Lilly and Company (Indianapolis,

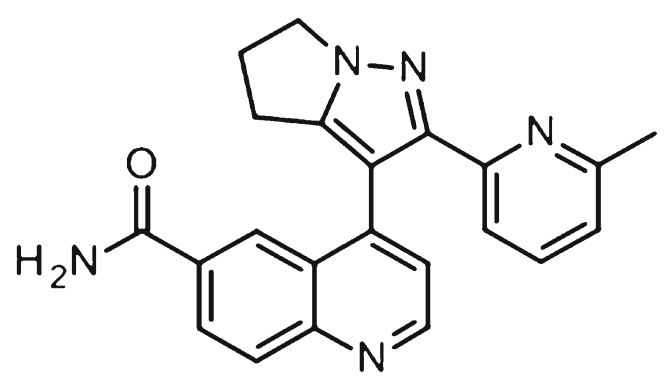

Fig. 1. Chemical structure of galunisertib (MW=369.4 Da)

IN, USA) for cancers. All other chemicals were purchased from commercial vendors and were of analytical grade. Human carcinoma of colon (Caco-2) cell line was purchased from the American Type Culture Collection (ATCC, Rockville, MD, USA).

\section{Solubility Measurement}

Equilibrium solubility of galunisertib in a crystalline form was measured in water, 0.01-0.1 N HCl, USP buffers, $0.1 \mathrm{~N}$ $\mathrm{NaOH}$, and simulated GI fluids. Fasted-state simulated gastric fluid (FaSSGF), fasted- or fed-state simulated intestinal fluids (FaSSIF and FeSSIF) were prepared as previously described (11).

Appropriate quantities of the compound were placed in glass vials, and $1 \mathrm{~mL}$ of each medium was added to achieve target concentration of $50 \mathrm{mg} / \mathrm{mL}$ in $\mathrm{HCl}$ solutions, and $2 \mathrm{mg}$ / $\mathrm{mL}$ in other media to maintain the buffering capacity. The vials were slowly rotated for $24 \mathrm{~h}$ at room temperature. Samples were then filtered without significant adsorption using Ultrafree MC centrifugal devices with a $0.22-\mu \mathrm{m}$ Durapore ${ }^{\circledR}$ PVDF membrane (EMD Millipore, Billerica, MA, USA), and the drug concentrations in solutions were determined by isocratic high-performance liquid chromatography (HPLC). The solution $\mathrm{pH}$ values were also measured. The HPLC system was composed of a Waters 2695 (Alliance) model with a UV detector. An Agilent Bonus RP column $(4.6 \times 75 \mathrm{~mm}, 3.5 \mu \mathrm{m})$ was used to analyze these samples. The injection volume was $2 \mu \mathrm{L}$. The mobile phase consisted of a mixture of acetonitrile $/ 0.1 \%$ trifluoroacetic acid and deionized water. The flow rate was $1.5 \mathrm{~mL} / \mathrm{min}$. The detector wavelength was set at $235 \mathrm{~nm}$.

\section{Permeability Assay}

Apparent permeability across the Caco-2 cell monolayers was determined following the current industrial practice (25). In summary, the assay was conducted after 21 days of cell growth to confluence on polystyrene membrane $(0.4 \mu \mathrm{m}$ pore size $)$ in 12 -well Corning® Transwell plates. Atenolol, propranolol, and digoxin were included in the study as references to classify permeability and evaluate active efflux. Galunisertib and reference compounds were dosed at $10 \mu \mathrm{M}$ in Hank's Balanced Salt Solutions (HBSS). Bi-directional transport was carried out in triplicate for galunisertib and in duplicate for reference compounds at $37^{\circ} \mathrm{C}$ for $2 \mathrm{~h}$. Galunisertib was diluted in HBSS to $10 \mu \mathrm{M}, \mathrm{pH}$ 6.5 for apical to basolateral transport, or $\mathrm{pH} 7.4$ for basolateral to apical transport. The apparent permeability 
value of galunisertib was determined by measuring the appearance rate of galunisertib in the receiver chambers across the transport period. The plates were stirred at $50 \mathrm{rpm}$ on an orbital shaker in the incubator at $37^{\circ} \mathrm{C}$. At every $30 \mathrm{~min}, 100 \mu \mathrm{L}$ samples were collected from the receiver chambers and replaced with equal volumes of blank HBSS. At the end of the transport, solutions in both donor and receiver chambers were collected to determine the recovery of galunisertib and reference compounds. Samples were analyzed using liquid chromatography with tandem mass spectrometry (LC/MS/MS) (26). Cell integrity throughout the transport was evaluated by transcellular epithelial electrical resistance (TEER) measurement and Lucifer yellow permeation test. TEER was measured before and immediately after the transport. The pre- and post-transport TEER must be above $300 \Omega \bullet \mathrm{cm}^{2}$, and the post-transport TEER must be at least $75 \%$ of the pre-transport values. The permeability of Lucifer yellow must not exceed $1 \times 10^{-6} \mathrm{~cm} / \mathrm{s}$.

\section{Clinical Product Development}

The drug solution used in Trial 1 was made by dissolving $150 \mathrm{mg}$ of radiolabeled galunisertib in $30 \mathrm{~mL}$ of $20 \%$ Captisol in $25 \mathrm{mM}$ phosphate buffer $(\mathrm{pH} 2)$. The original galunisertib tablet formulation consisted of a high shear wet granulation (HSWG) process involving tray drying and tablet compression with no film coating. Three tablet strengths were developed: 10,50 , and $100 \mathrm{mg}$ tablets to accommodate flexible dosing in early clinical trials. The $50 \mathrm{mg}$ HSWG tablets were used in dissolution tests and Trial 2 (dosed as $3 \times 50 \mathrm{mg}$ ). To support potential commercialization, a roller compaction (RC) manufacturing process and unit composition was developed later. The new manufacturing process consisted of standard tableting excipients. Materials were blended, roller compacted, and then mixed with extragranular powders and compressed to produce core tablets of the desired drug content (i.e., $150 \mathrm{mg}$ as a projected clinical efficacious dose). The core tablets were coated with soluble film. Two different batches of drug substance were used in these formulations. The early batch was processed by dry milling which resulted in equivalent diameters of $50 \mu \mathrm{m}$ or less for $90 \%$ of the drug particles. The late batch was processed by slurry milling which resulted in diameters of $80 \mu \mathrm{m}$ or less for $90 \%$ of the drug particles. The dry-milled batch was formulated into HSWG and some RC tablets (designated as RCD in Trial 2), while the slurry-milled batch was used in the remaining RC tablets (designated as RCS in Trial 2).

\section{USP Dissolution Test}

In vitro dissolution rates were determined for the tablet formulations used in the clinical study. The media included $0.01 \mathrm{~N} \mathrm{HCl}$ ( $\mathrm{pH} 2), 50 \mathrm{mM}$ citrate/phosphate buffer ( $\mathrm{pH} 3.5)$, $50 \mathrm{mM}$ acetate buffer ( $\mathrm{pH} 4.5)$, and $50 \mathrm{mM}$ phosphate buffer (pH 6.8). Dissolution rates were measured as per USP $<905>$ using the paddle apparatus at $75 \mathrm{rpm}$, and $900 \mathrm{~mL}$ of each media (Distek Evolution 6100 for $\mathrm{pH} 2$ and 3.5, Sotax AT70 with Sotax CP7 autosampler for $\mathrm{pH} 4.5$ and 6.8). The amount of drug in solution as a function of time was determined by UV detection at a wavelength of $275 \mathrm{~nm}$ for
pH 2, and $308 \mathrm{~nm}$ for all other pH (Leap Technologies OPTDISS UV fiber optic with $5 \mathrm{~mm}$ path length probes for $\mathrm{pH} 2$ and $1 \mathrm{~mm}$ path length probes for $\mathrm{pH} 3.5$, Agilent 8453 with $1 \mathrm{~mm}$ path length flow cell for both $\mathrm{pH} 4.5$ and 6.8). At $\mathrm{pH}$ 3.5 and above, three unit doses were placed in each vessel for the $50 \mathrm{mg}$ HSWG formulation in order to provide equivalent concentration to the $150 \mathrm{mg} \mathrm{RC}$ formulations.

\section{Artificial Stomach-Duodenum Dissolution}

The artificial stomach-duodenum (ASD) model was used to simulate physiological $\mathrm{pH}$ and mass transfer conditions in order to measure the dissolution behavior of the compound in different formulations. Its basic construction has been reported previously (18). Specifically for this experiment, the setup comprises three compartments-the stomach chamber, duodenum chamber, and overflow. All of these were jacketed vessels maintained at $37^{\circ} \mathrm{C}$ using a circulating water bath. The stomach chamber contained $50 \mathrm{~mL}$ of $0.01 \mathrm{~N} \mathrm{HCl} / 0.034 \mathrm{M}$ $\mathrm{NaCl}$ plus $200 \mathrm{~mL}$ of DI water. The duodenum chamber contained $30 \mathrm{~mL}$ of FaSSIF. Fresh FaSSIF was pumped into the "duodenum" continuously at $2 \mathrm{~mL} / \mathrm{min}$ at $37^{\circ} \mathrm{C}$. A computer-controlled transfer pump transferred the stomach chamber contents into the duodenum chamber in a first-order fashion with a half-life of $30 \mathrm{~min}$. The volume of the duodenum chamber was kept constant at $30 \mathrm{~mL}$ using a vacuum line. The removed contents from the duodenum were collected in the overflow chamber. In situ absorbance and $\mathrm{pH}$ measurements were obtained using the Rainbow DDMS with Pion fiber optic probes placed in all three compartments to collect real time data during the experiment. The duration of the experiment was $150 \mathrm{~min}$.

\section{Preclinical Animal Study}

The preclinical study was conducted in fasted male F344 rats to determine the oral bioavailability of galunisertib. Animal protocols were approved and carried out under Eli Lilly Animal Care and Use Committee guidelines. Male rats $(N=3)$ were administered a single oral dose at $10 \mathrm{mg} / \mathrm{kg}$ and then a bolus IV dose at $1 \mathrm{mg} / \mathrm{kg}$ of galunisertib. The doses were dissolved in $1 \mathrm{~mL} / \mathrm{kg}$ of saline acidified with $1 \mathrm{~N} \mathrm{HCl}$ for IV administration, and suspended in $10 \mathrm{~mL} / \mathrm{kg}$ of $1 \%$ carboxymethylcellulose, $0.25 \%$ polysorbate 80 , and $0.05 \%$ antifoam 1510-US for oral administration. Venous blood samples were serially collected at several time points after each dose in heparinized tubes, and plasma concentrations of galunisertib were determined by LC/MS/MS.

\section{Clinical Studies}

Trial 1 was a single-center, open-label study, conducted to determine the disposition of radioactivity and metabolism of galunisertib in healthy subjects following oral administration of a single dose of $150 \mathrm{mg}$ (approximately $100 \mu \mathrm{Ci}$ ) $\left[{ }^{14} \mathrm{C}\right]-$ galunisertib, in surgically sterile or postmenopausal healthy females, or sterile healthy males. Six subjects received a single oral dose of $150 \mathrm{mg}$ galunisertib, administered as an oral solution. Venous blood samples (approximately $10 \mathrm{~mL}$ ) were collected for pharmacokinetic (PK) analysis and radioanalysis. Whole blood and plasma samples were used 
to determine total radioactivity, and plasma samples were used to determine concentrations of galunisertib. Samples were collected at pre-dose, 0.5, 1, 2, 3, 4, 6, 8, 10, 12, 24, 48, $72,96,120$, and $144 \mathrm{~h}$ post-dose, and every $24 \mathrm{~h}$ thereafter until the specified release criteria had been met. The samples were analyzed for galunisertib using validated liquid chromatography atmospheric pressure ionization tandem mass spectrometry methods where the lower limit of quantification was $5.00 \mathrm{ng} / \mathrm{mL}$ and the upper limit of quantification was $1000.00 \mathrm{ng} / \mathrm{mL}$. The inter-assay accuracy (\% relative error) during validation ranged from -2.22 to $-1.79 \%$. The interassay precision ( $\%$ relative $\mathrm{SD})$ during validation ranged from 2.21 to $5.07 \%$.

Trial 2 was a relative bioavailability evaluation of three formulations of galunisertib administered as a monotherapy in patients with advanced or metastatic cancer. This was an open-label, single-center, 3-period crossover, relative bioavailability (RBA) study. Each sequence had three periods, with patients being administered each formulation at a single dose of $150 \mathrm{mg}$ orally (three 50-mg HSWG tablets were dosed as opposed to one 150-mg RCD or RCS tablet) on the first day of each period. Patients were enrolled in sets of 6 in order that all 6 sequences had the same number of completers. Samples were collected for PK analysis and metabolite profiling up to $48 \mathrm{~h}$ post-dose of each period. The samples were analyzed for galunisertib using two validated liquid chromatography coupled with tandem mass spectrometry methods. For the high-range method, the lower limit of quantification (LLOQ) was $5.00 \mathrm{ng} / \mathrm{mL}$, and the upper limit of quantification was $1000.00 \mathrm{ng} / \mathrm{mL}$. The intra-assay accuracy (\% relative error) during validation ranged from 1.80 to $10.22 \%$. The intra-assay precision ( $\%$ relative standard deviation) during validation ranged from 3.12 to $18.39 \%$. For the low-range method, the LLOQ was $0.050 \mathrm{ng} / \mathrm{mL}$, and the upper limit of quantification was $10.00 \mathrm{ng} / \mathrm{mL}$. The intraassay accuracy during validation ranged from -5.08 to $-2.00 \%$. The intra-assay precision during validation ranged from 4.40 to $12.25 \%$. Galunisertib is stable in human plasma for up to 567 days when stored at approximately $-70^{\circ} \mathrm{C}$ and up to 148 days when stored at approximately $-20^{\circ} \mathrm{C}$ for the low-range method.

\section{Oral Absorption Modeling}

Preclinical and clinical PK studies were simulated using GastroPlus ${ }^{\circledR}$ (v.8.0) with focus on understanding the in vivo absorption of galunisertib and its sensitivity to inputs associated with compound properties or formulation factors. GastroPlus is a commercial program developed by Simulations Plus Inc. (Lancaster, CA, USA). It uses an advanced compartmental absorption and transit model to simulate drug absorption relative to its dissolution and transit in the GIT (27). The primary utility of simulating the rat bioavailability study result was to estimate the in vivo effective permeability $\left(\mathrm{P}_{\text {eff }}\right)$ and establish a base absorption model. Compartmental PK parameters in rats were estimated from mean plasma concentration-time profile after IV administrations using the PKPlus module within GastroPlus. By fitting the measured solubility data based on the Henderson-Hasselbalch equation, GastroPlus constructed a pH-solubility profile that could calculate solubility of galunisertib in the $\mathrm{pH}$ range of 1-8.
Precipitation time was calculated by GastroPlus based on ASD results (The calculation is described in details together with the result of ASD tests in "RESULTS"). Drug particle size in the dosing suspension was assumed as small as $10 \mu \mathrm{m}$ in radius to minimize its impact on dissolution. As for other absorption parameters that were not readily available experimentally, we took the calculation by GastroPlus (e.g., diffusion coefficient), or the default options (e.g., ASF model). By fitting the observed mean plasma concentrationtime profile after oral administration, we obtained the appropriate value for permeability.

As concomitant IV administration is not routinely conducted during early clinical studies, to identify an appropriate clinical PK model, we simulated mean plasma concentration-time profile observed with healthy subjects in Trial 1 independently by two approaches, i.e., compartmental versus physiologically based PK (PBPK) modeling. Both PK models were coupled with the base absorption model that was established from simulation of the rat study. The compartmental PK model employed similar approach reported previously. Briefly, we leveraged the noncompartmental analysis of the observed concentration-time profile and the Optimization module in GastroPlus to identify a twocompartmental PK model and estimate corresponding PK parameters for galunisertib. The drug was predominantly cleared by oxidative metabolism. A substrate depletion approach using recombinant human cytochromes $\mathrm{P} 450$ (rCYPs) concluded the hepatic CYP-mediated clearance was primarily through CYP3A4, further confirmed by the ${ }^{14} \mathrm{C}$ study (i.e., Trial 1). Hence, we also utilized the in vitro human liver microsomal and rCYP clearance data to extrapolate the in vivo systemic clearance (CL), assuming a well-stirred model of hepatic clearance. Assuming the distribution of galunisertib through the tissues was primarily limited by perfusion, we estimated the tissue partitioning coefficient Kp's with various methods provided by GastroPlus (28-30), in order to achieve the best fit of the observed PK profile. The hepatic extraction ratio $E_{h}$ was estimated by Eq. 1 .

$E_{\mathrm{h}}=\frac{\mathrm{CL}}{Q_{\mathrm{h}}}$

where $Q_{\mathrm{h}}$ is the hepatic blood flow. The gut extraction ratio $E_{\mathrm{g}}$ was calculated from Eqs. 2 and 3

$F=F_{\mathrm{a}} \times\left(1-E_{\mathrm{g}}\right) \times\left(1-E_{\mathrm{h}}\right)$

$F=\frac{\mathrm{AUC}}{\mathrm{Dose}} \times \mathrm{CL}$

$F_{\text {a }}$, i.e., fraction of dose absorbed, was calculated from the base absorption model, which was suggested to be $100 \%$ in Trial 1.

After completing a whole PK model that could describe ADME processes of galunisertib in human, we incorporated formulation factors to construct a biopharmaceutical model for the prediction of formulation effect on the absorption of 
galunisertib. GastroPlus provides several dissolution models to estimate in vivo dissolution rates and construct dose dissolved versus time profiles. The Johnson model (31) is the default option which we used to simulate in vivo redissolution of the drug administered in solution in Trial 1 . To integrate the in vitro USP dissolution data obtained for the three drug products administered in Trial 2, we selected z-factor dissolution model to evaluate potential formulation effect on their in vivo dissolution and absorption (32). The so-called dissolution parameter $z$ is defined in the equation below,

$\frac{\mathrm{dA}_{\mathrm{d}}}{\mathrm{dt}}=\frac{3 D}{\rho h r} \times A_{0}\left(\frac{A_{\mathrm{s}}}{A_{0}}\right)^{2 / 3}\left(C_{\mathrm{s}}-\frac{A_{\mathrm{d}}}{V}\right)$

where $A_{\mathrm{d}}$ is the amount of drug dissolved in the media, $A_{\mathrm{s}}$ the amount of undissolved solid drug in the media, $A_{0}$ the initial amount of drug dosed in the media, $C_{\mathrm{s}}$ the solubility of the drug in the media, and $V$ the volume of the media. Z, i.e., $\frac{3 D}{\rho h r}$ in Eq. 4, is a hybrid parameter of the size of assumedly spherical particles $r$, the diffusion coefficient $D$, density of the $\operatorname{drug} \rho$, and the diffusion layer thickness $h$. It is independent of the solubility, dose, and the volume of medium. The $z$ values were estimated from in vitro dissolution data by GastroPlus, and used subsequently in conjunction with variable solubility and drug concentrations during the transit in different regions of the GIT to construct the in vivo dissolution profiles. In this case, we assigned two discreet $z$ values (estimated from test results at $\mathrm{pH} 2$ and 6.8) as approximation of $z$ 's in gastric and intestinal dissolution. This biopharmaceutical model, composed of z-factor dissolution and passive permeation under typical fasted human physiology, was applied with the PK model for cancer patients to predict the in vivo performances of the immediate-release tablets of galunisertib. The predictability of these models was evaluated by comparing area under the plasma concentration versus time curve (AUC), $\mathrm{C}_{\max }, \mathrm{T}_{\max }$, and overall shape of the PK profiles between observation and prediction.

\section{RESULTS}

\section{In vitro Absorption Properties}

The drug properties that determine its absorption are solubility and permeability in the GIT. Galunisertib was measured with two basic pKa's of 4.3 and 2.8. Consistent with its ionization potential, its aqueous solubility is sensitive to $\mathrm{pH}$, manifested by solubility of $20 \mathrm{mg} / \mathrm{mL}$ or higher at $\mathrm{pH}<3$ and a significant reduction to $0.05 \mathrm{mg} / \mathrm{mL}$ at $\mathrm{pH}>6$. The solubility values in the simulated fluids (i.e., $0.05 \mathrm{mg} / \mathrm{mL}$ in FaSSIF at pH 6.5 and $0.12 \mathrm{mg} / \mathrm{mL}$ FeSSIF at $\mathrm{pH} 5.0$ ) are comparable to those in aqueous buffers at similar pH's, indicating minimal solubilization by bile salts particularly in the fasted state. A good fit of $\mathrm{pH}$-solubility profile using Henderson-Hasselbalch equation was achieved and presented in Fig. 2. Human effective permeability $\left(\mathrm{P}_{\mathrm{eff}}\right)$ of galunisertib was calculated as $1.5 \times 10^{-4} \mathrm{~cm} / \mathrm{s}$, using an in-house QSAR model (33). With sufficient recovery (>90\%) and wellmaintained cellular integrity, apparent permeability $\left(\mathrm{P}_{\mathrm{app}}\right)$ of galunisertib across the Caco- 2 cell monolayers was estimated at $(3.5 \pm 0.25) \times 10^{-6} \mathrm{~cm} / \mathrm{s}$ from the apical to basolateral side,

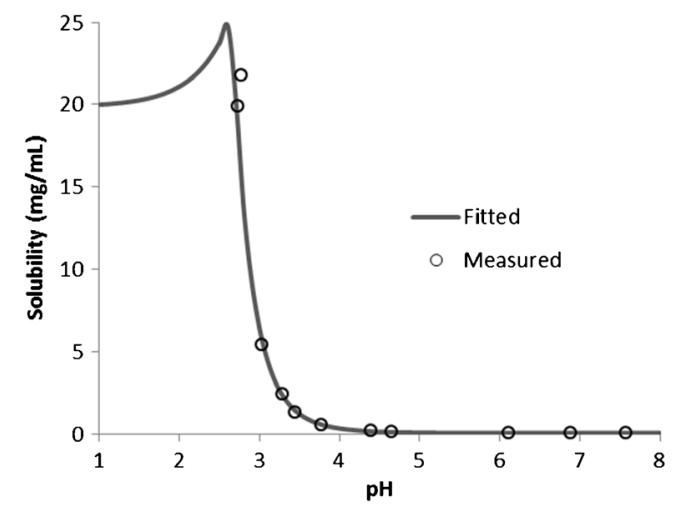

Fig. 2. Solubility-pH profile of galunisertib fitted with HendersonHasselbalch equation by GastroPlus. In vitro measurements are in the range of $0.05-21.8 \mathrm{mg} / \mathrm{mL}$ from $\mathrm{pH} 2.7-7.6$

and $(12.2 \pm 0.38) \times 10^{-6} \mathrm{~cm} / \mathrm{s}$ from the basolateral to apical side. The polarized transport, manifested by an efflux ratio of 3.5, implied that galunisertib is likely a substrate of $\mathrm{P}$-glycoprotein (P-gp). However, as compared to digoxin that had an efflux ratio of 15 in this assay, galunisertib is presumably a weak substrate. The apical $\mathrm{P}_{\mathrm{app}}$ of the drug was approximately $3 \times$ lower than that of propranolol, $2 \times$ as high as that of atenolol, and $5 \times$ as high as that of digoxin. The Caco- 2 assay was designed primarily for qualitative classification of drug permeability in the GIT and assessment of P-gp efflux, rather than quantitative determination of permeability since no correlation model between $\mathrm{P}_{\text {app }}$ and $\mathrm{P}_{\text {eff }}$ was attempted. Nevertheless, based on the human $\mathrm{P}_{\text {eff }}$ values of these reference compounds (2), $\mathrm{P}_{\text {eff }}$ of galunisertib can be assigned in the range of $(0.2-2.9) \times 10^{-4} \mathrm{~cm} / \mathrm{s}$. The calculated $\mathrm{P}_{\text {eff }}$ falls in this range. It is well documented that $\mathrm{pH}$ may affect permeability through ionization effect $(34,35)$. Such $\mathrm{pH}-$ effect may contribute to the difference between bidirectional $\mathrm{P}_{\text {app }}$ 's if using $\mathrm{pH}$-gradient across the monolayers to mimic the physiological condition of the GIT. This effect, however, is likely minimal in this case as a majority of galunisertib molecules are in neutral form at $\mathrm{pH} 6.5$ or 7.4. The drug itself demonstrated sufficient chemical stability in aqueous solutions or as solids under physiologically relevant conditions (e.g., temperature, $\mathrm{pH}$, duration, etc.).

\section{USP Dissolution Test}

In vitro tablet dissolution results are shown in Fig. 3. Results are plotted as percent of drug released versus time in minutes. The axis for plots $\mathrm{C}$ and $\mathrm{D}$ are different from $\mathrm{A}$ and $\mathrm{B}$ in order to clearly show the differences in rate between formulations at each $\mathrm{pH}$ condition. In $0.01 \mathrm{~N} \mathrm{pH} 2 \mathrm{HCl}$ (Plot A), dissolution is rapid due to very high solubility (>20 mg/ $\mathrm{mL}$ ). Visually, it was observed that the HSWG tablets were slower to disintegrate, approximately $5 \mathrm{~min}$, compared to the RCD and RCS tablets, which disintegrated in about $1 \mathrm{~min}$. The difference in dissolution rate at $\mathrm{pH} 2$ appears to be primarily driven by tablet disintegration time.

In the pH 3.5 medium (Plot B), dissolution of the HSWG is observed to be faster than the RCD and RCS tablets. In this medium, the tablet disintegration times were visually observed to be similar to those observed at $\mathrm{pH} 2.0$ ( $\sim 5 \mathrm{~min}$ for HSWG, and $\sim 1$ min for RCS and RCD). At $\mathrm{pH} 3.5$, the drug substance 

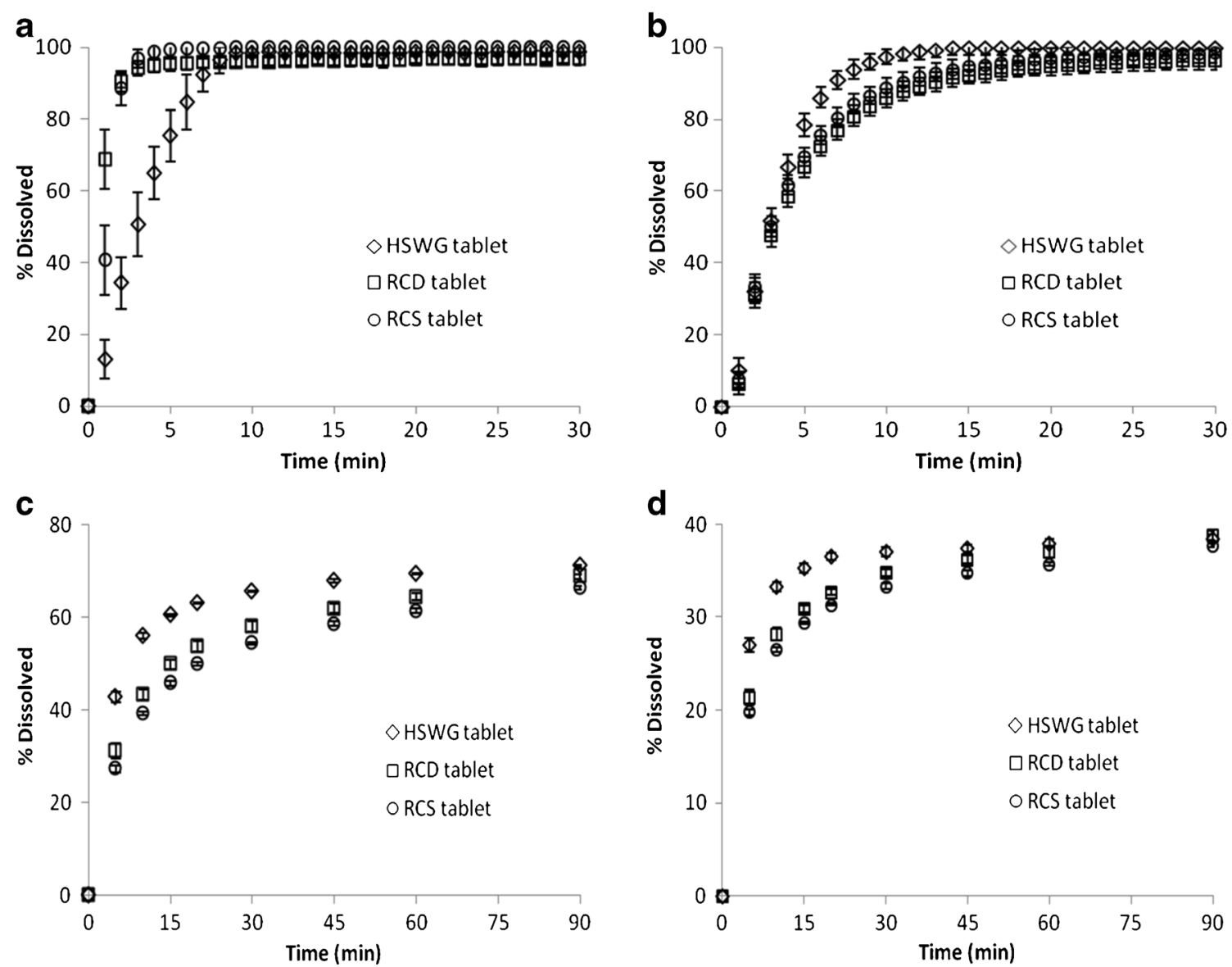

Fig. 3. In vitro dissolution profiles of galunisertib tablets. a In $0.01 \mathrm{~N} \mathrm{HCl}$ at $\mathrm{pH}$ 2. b In citrate phosphate buffer at $\mathrm{pH} 3.5$. c In acetate phosphate buffer at $\mathrm{pH} 4.5$. $\mathbf{d}$ In phosphate buffer at $\mathrm{pH} 6.8$

solubility is more limiting $(1.3 \mathrm{mg} / \mathrm{mL})$ and is approximately 8 times the solubility needed to dissolve the 150 -mg dose. Since the drug substance particle size is similar for HSWG and RCD, it is hypothesized that the differences in dissolution rate for these tablets in this media are driven by differences in the dissolution of granules created by high-sheer granulation compared to those from roller compaction processes.

At $\mathrm{pH} 4.5$ and 6.8 (plots $\mathrm{C}$ and $\mathrm{D}$ ), dissolution is solubility limited, plateauing at approximately $70 \%$ (i.e., $105 \mathrm{mg}$ ) released at $\mathrm{pH} 4.5$ and approximately $35 \%(53 \mathrm{mg})$ at $\mathrm{pH} 6.8$, consistent with the solubility measured as $0.12 \mathrm{mg}$ / $\mathrm{mL}$ and $0.05 \mathrm{mg} / \mathrm{mL}$, respectively. Similar to the result observed at $\mathrm{pH} 3.5$, the HSWG tablets show faster dissolution compared to the RCD and RCS tablets. Again, this difference in rate is believed to be due to differences in granule dissolution as a function of granulation process. While the RCD and RCS tablets are manufactured with different particle size drug substance, little difference in dissolution rate is observed between them at any $\mathrm{pH}$. This further supports the hypothesis that granule dissolution is rate limiting.

\section{ASD Dissolution}

The results of this dynamic dissolution test are presented in Fig. 4 and Table I. The concentration-time profiles in the stomach and duodenum chambers are determined by kinetics of dissolution, precipitation, and transit of galunisertib. The theoretical duodenal concentration-time profile was constructed by assuming no precipitation of galunisertib in the duodenum. Therefore, the profile was determined only by input of dissolved drug from the stomach and outflow of the drug solution. In this case, we constructed the theoretical profile for galunisertib in the solution formulation based on the method reported previously (36). By comparison of the actual experimental profiles to the theoretical one, we could evaluate the occurrence of precipitation which may be indicated by reduced duodenal peak concentrations and/or exposures.

In the "stomach," the initial concentration increase occurring to the tablets indicates dissolution of galunisertib after the observed rapid disintegration of tablets; decline of the concentration starting around 10$15 \mathrm{~min}$ is due to the transfer of galunisertib to the "duodenum." Concentrations of galunisertib reached the peaks at approximately the same time for all three tablets; however, HSWG tablet achieved apparently higher $\mathrm{C}_{\max }$ and AUC than RC tablets in the "stomach." In the "duodenum," solution formulation achieved peak concentration at earlier time than tablets; however, highest $\mathrm{C}_{\max }$ and AUC occurred to HSWG tablets. In spite of slightly higher $\mathrm{C}_{\max }$ by solution formulation than $\mathrm{RC}$ tablets, their 


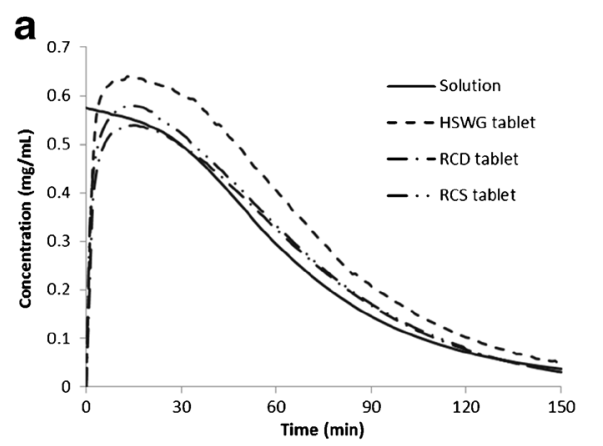

b
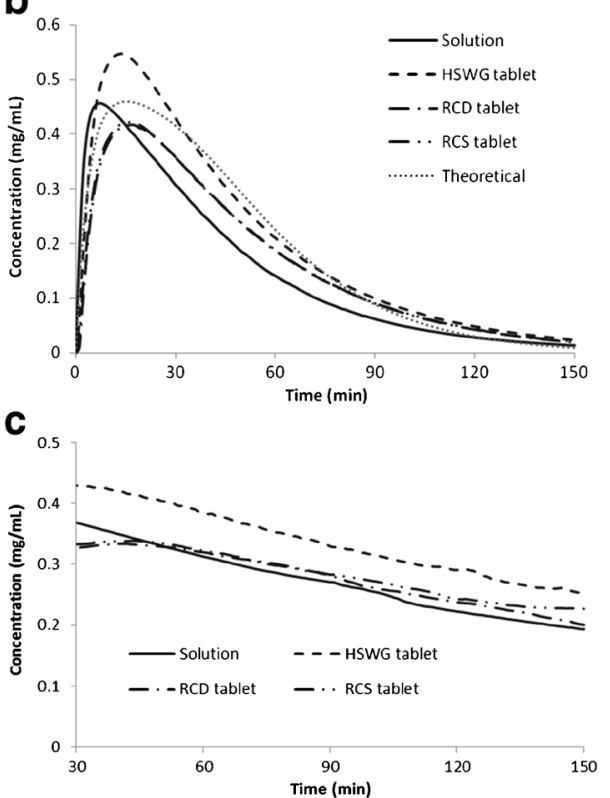

Fig. 4. Concentration-time profiles of galunisertib in solution or tablets at $150 \mathrm{mg}$ in ASD dissolution tests. a Gastric chamber. b Duodenal chamber. c Overflow

AUC's are similar in the duodenum. The solubility of galunisertib in the acidic "stomach" is adequate for rapidly dissolving and/or maintaining $150 \mathrm{mg}$ dose in solution. Upon entry to the "duodenum," solubility is significantly decreased at $\mathrm{pH} \geq 6$ and becomes insufficient to maintain the dose in solution, potentially leading to precipitation of galunisertib in the "small intestine" (represented by the duodenum and overflow chambers). The gradual decline of concentration in the overflow over the test time is attributed to dilution by the continuous pumping of fresh fluid in the "duodenum," and possible precipitation. However, comparable duodenal concentrations and exposure between experimental and theoretical profiles implied no significant precipitation might have occurred.

Over the test time, $\mathrm{pH}$ in the "stomach" increased to 3.2 presumably due to the ionization of the dissolved basic drug; but $\mathrm{pH}$ in the "small intestine" was maintained around 6.5. Based on the equilibrium solubility measurements, galunisertib shall reach a saturated concentration of $0.05 \mathrm{mg} / \mathrm{mL}$ at $\mathrm{pH} 6.5$. Nevertheless the drug maintained concentrations of about $0.2 \mathrm{mg} / \mathrm{mL}$ in all the formulations in the overflow at the end of test. Since the overflow collected a majority of fluid from the "stomach" and "duodenum," we estimated approximately $120 \mathrm{mg}$ or more of galunisertib should remain dissolved at $150 \mathrm{~min}$ of dosing, indicating galunisertib may effectively maintain supersaturation in the upper small intestine. This quantitative result is consistent with the qualitative comparison between experimental and theoretical profiles. To leverage this important finding in the absorption modeling, we estimated the precipitation time for GastroPlus input. We simulated dissolution-precipitation of the drug in GastroPlus by turning off permeation (i.e., setting $\mathrm{P}_{\text {eff }}=0$ in this exercise). To maintain $120 \mathrm{mg}$ of dose dissolved at 150 min of transfer in the GIT, the precipitation time was estimated as approximately $11 \mathrm{~h}(40,000 \mathrm{~s}$ as the input value).

\section{Preclinical Animal Study and Simulation}

The mean bioavailability of galunisertib was estimated to be $69 \%$ in rats based upon AUC comparison at an oral and IV dose of 10 and $1 \mathrm{mg} / \mathrm{kg}$. After oral administration, galunisertib reached maximum plasma concentration rapidly in $0.5 \mathrm{~h}$. The moderately high bioavailability and quick onset imply rapid and high absorption of galunisertib in the rodent GIT. As shown in Fig. 5, simulation of the plasma concentration-time profile suggested complete absorption $\left(100 \% F_{a}\right)$ of galunisertib dosed as an oral suspension at $10 \mathrm{mg} / \mathrm{kg}$ in rats. A parameter sensitivity analysis suggested $P_{\text {eff }}$ has to be $0.64 \times 10^{-4} \mathrm{~cm} / \mathrm{s}$ or higher in rats to achieve $80 \%$ of the observed $\mathrm{C}_{\max }$ value. GastroPlus adopted a simple scaling to calculate human $\mathrm{P}_{\mathrm{eff}}$ from rat or vice versa, based on the correlation of $\mathrm{P}_{\text {eff }}$ between these species established from perfusion results. Using the scalar between human and rat implemented by GastroPlus, we estimated human $\mathrm{P}_{\text {eff }}$ as $1.77 \times 10^{-4} \mathrm{~cm} / \mathrm{s}$ at a minimum, which is similar to the calculation from the in-house QSAR model.

Table I. ASD Dissolution Profile Parameters for $150 \mathrm{mg}$ Galunisertib Obtained in the Fasted State

\begin{tabular}{|c|c|c|c|c|c|c|}
\hline \multirow[b]{2}{*}{ Category } & \multicolumn{2}{|c|}{$\mathrm{AUC}(\mathrm{mg} \times \min / \mathrm{mL})$} & \multicolumn{2}{|c|}{$C_{\max }(\mathrm{mg} / \mathrm{mL})$} & \multicolumn{2}{|c|}{$T_{\max }(\min )$} \\
\hline & Stomach & Duodenum & Stomach & Duodenum & Stomach & Duodenum \\
\hline Solution & 39.4 & 24.66 & 0.57 & 0.46 & 0 & 7 \\
\hline HSWG tablet & 48.8 & 30.48 & 0.64 & 0.55 & 13 & 14 \\
\hline RCD tablet & 39.6 & 25.19 & 0.54 & 0.42 & 13 & 17 \\
\hline RCS tablet & 41.4 & 25.35 & 0.58 & 0.42 & 14 & 16 \\
\hline Theoretical & ND & 28.48 & ND & 0.46 & ND & 14 \\
\hline
\end{tabular}




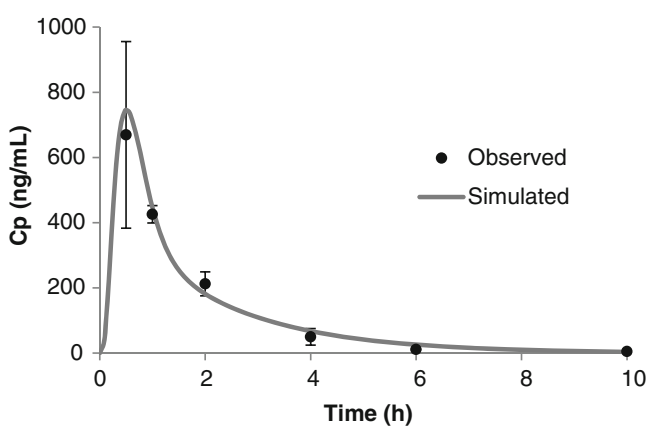

Fig. 5. Observed and simulated mean plasma concentration-time profile of galunisertib in rats after oral administration of $10 \mathrm{mg} / \mathrm{kg}$ in suspension

\section{Clinical Study Results}

In Trial 1 a total of 6 healthy subjects, 4 males and 2 females, between the ages of 31 to 60 years with a BMI range of 23.6 and $30.3 \mathrm{~kg} / \mathrm{m}^{2}$ participated. Table II presents the geometric PK parameters for galunisertib following a single 150 -mg dose of galunisertib containing approximately $100 \mu \mathrm{Ci}$ of $\left[{ }^{14} \mathrm{C}\right]$-galunisertib. The plasma concentration versus time profiles were characterized by a rapid absorption phase, with $T_{\max }$ observed at $0.50 \mathrm{~h}$ in all subjects. Following attainment of $C_{\max }$, plasma concentrations of galunisertib appeared to decline in a biphasic manner with a potential tertiary elimination phase observed in some subjects where galunisertib concentrations were quantifiable at sampling times later than $48 \mathrm{~h}$ post-dose. Galunisertib was quantifiable in plasma for up to $48 \mathrm{~h}$ post-dose for 2 subjects, up to $72 \mathrm{~h}$ post-dose for 2 subjects, up to $96 \mathrm{~h}$ post-dose for 1 subject, and up to $144 \mathrm{~h}$ post-dose for 1 subject. The percentage of $\mathrm{AUC}_{(0-\infty)}$ extrapolated was low, with $\% \mathrm{AUC}_{(\text {tlast- } \infty)}$ ranging from 7 to $12 \%$ for plasma. The between-subject variability in the $\mathrm{PK}$ of plasma galunisertib was moderate, with the geometric coefficient of variation
$\left(\mathrm{CV} \%\right.$ ) for $\mathrm{AUC}_{(0-12)}, \mathrm{AUC}_{(0-\infty)}$, and $C_{\max }$ being 35,36 , and $37 \%$, respectively.

In Trial 2, a total of 14 patients were enrolled in the study, of which 9 (64\%) were female, and all (100\%) were white. The mean age was 59.8 years (range, 34 to 76 years), and mean body mass index was $26.3 \mathrm{~kg} / \mathrm{m}^{2}$ (range, 18.8 to $41.5 \mathrm{~kg} / \mathrm{m}^{2}$ ). The majority of patients had a baseline Eastern Cooperative Oncology Group performance status of 1 (8 patients; $57 \%$ ). The analysis results on all subjects (Table II) show that, on average, exposures (as determined by $\mathrm{AUC}_{(0-}$ tlast) and $\left.\mathrm{AUC}_{(0-\infty)}\right)$ were comparable, but $C_{\max }$ values were reduced by approximately 23 and $20 \%$ for the RCD and RCS formulations, respectively, compared to the HSWG formulation.

\section{Clinical Study Simulations}

Table III summarized the key inputs for the absorption models that were used to describe the absorption of galunisertib in the trials. The base model, established from in vitro experimental data and simulation of preclinical study results, as described previously, was directly applied in the simulation of the plasma concentration-time profile of galunisertib dosed in solution. Similar estimation of systemic CL (i.e., 24.5, 25.9, and 29.2 L/h) was obtained from fitting the profile by GastroPlus using compartmental PK modeling, IVIVE (in vitro-in vivo extrapolation) from human liver microsomes or $\mathrm{rCYP} 3 \mathrm{~A}$, respectively. PBPK models that were built from various $K_{p}$ calculation methods could not produce any reasonable fit for the profile, e.g., predicted $C_{\text {max }}$ 's were all less than $50 \%$ of the observed value. Therefore, a two-compartmental PK model was believed to be sufficient and accepted to simulate all the clinical results reported here. As shown in Fig. 6, simulation of the mean concentration-time profile in Trial 1 using this base absorption and compartmental PK models agrees well with the clinical observations.

Table II. Galunisertib Noncompartmental Pharmacokinetic Parameters Following a Single Oral Dose of Galunisertib Given as Solution (Trial 1) or Either High-Sheer Wet Granulation, Roller Compaction Dry-Milled, or Roller Compaction Slurry-Milled Formulations (Trial 2)

\begin{tabular}{|c|c|c|c|c|}
\hline Parameter $^{\mathrm{a}}$ & Trial 1 & Trial 2 & & \\
\hline Dose (mg) & $150(N=6)$ & $150(N=14)$ & $150(N=14)$ & $150(N=14)$ \\
\hline Formulation & solution & HSWG & $\mathrm{RCD}$ & RCS \\
\hline$T_{\max }(\mathrm{h})^{\mathrm{b}}$ & $0.50(0.50-0.50)$ & $1.00(0.50-3.08)$ & $2.00(0.50-3.17)$ & $2.00(0.50-3.00)$ \\
\hline$C_{\max }(\mu \mathrm{g} / \mathrm{L})$ & $1480(37)$ & $954(89.7)$ & $734(68.0)$ & $769(67.8)$ \\
\hline $\operatorname{AUC}_{(0 \text {-tlast })}(\mu \mathrm{g} \cdot \mathrm{h} / \mathrm{L})$ & $3660(36)$ & $4520(58.5)$ & $4360(52.0)$ & $4350(63.9)$ \\
\hline $\operatorname{AUC}_{(0-\infty)}(\mu \mathrm{g} \cdot \mathrm{h} / \mathrm{L})$ & $3670(36)$ & $4740(55.6)$ & $4490^{\mathrm{c}}(53.3)$ & $4790^{\mathrm{d}}(70.9)$ \\
\hline $\mathrm{CL} / \mathrm{F}(\mathrm{L} / \mathrm{h})$ & $40.9(36)$ & $31.7(55.6)$ & $33.4^{\mathrm{c}}(53.3)$ & $31.3^{\mathrm{d}}(70.9)$ \\
\hline $\mathrm{V}_{\mathrm{z}} / \mathrm{F}(\mathrm{L})$ & $508(53)$ & $505(88.0)$ & $473^{\mathrm{c}}(76.0)$ & $511^{\mathrm{d}}(81.9)$ \\
\hline $\mathrm{T}_{1 / 2}(\mathrm{~h})$ & $8.61(4.78-19.6)^{\mathrm{b}}$ & $11.1(47.0)$ & $9.81^{\mathrm{c}}(41.1)$ & $11.3^{\mathrm{d}}(42.4)$ \\
\hline
\end{tabular}

$A U C$ area under the plasma concentration versus time curve, $A U C_{(0-\infty)}$ AUC from zero to infinity, $A U C\left(0-t_{\text {last }}\right)$ AUC from time zero to time $\mathrm{t}$ where $\mathrm{t}$ is the last time point with a measurable concentration, $C L / F$ clearance, $C_{\max }$ maximum plasma drug concentration, $C V$ coefficient of variation, $H S W G$ high-sheer wet granulation, $N$ number of subjects used in pharmacokinetic analysis, $R C D$ roller compaction dry-milled, $R C S$ roller compaction slurry-milled, $t_{1 / 2}$ half-life associated with the terminal rate constant in noncompartmental analysis, $T_{\text {max }}$ time of maximum plasma drug concentration, $V_{z} / F$ volume of distribution

${ }^{a}$ All pharmacokinetic parameters single dose at Day 1 (geometric mean [\% CV] unless stated otherwise)

${ }^{b}$ Median (range)

${ }^{c} N=12$

${ }^{d} N=11$ 
Table III. Physiologically Based Absorption Models Used in GastroPlus Simulations

\begin{tabular}{ll}
\hline \multicolumn{1}{c}{ Parameter } & \multicolumn{1}{c}{ Value } \\
\hline Dosage form & IR: solution or tablet \\
LogP & 1.85 \\
Permeability $\left(\times 10^{-4} \mathrm{~cm} / \mathrm{s}\right)$ & 1.5 (in silico calculation, verified by in vivo rat data) \\
Solubility $(\mathrm{mg} / \mathrm{mL})$ & pH-solubility profile fitted to measured data by GastroPlus (see Fig. 2 for more details) \\
In vivo dissolution & Johnson model for redissolution of precipitate in solution; z-factor dissolution model for tablets \\
Diffusion coefficient $\left(\times 10^{-5} \mathrm{~cm}^{2} / \mathrm{s}\right)$ & 0.69 (calculated by GastroPlus based on MW) \\
Density $(\mathrm{g} / \mathrm{mL})$ & 1.2 (GastroPlus default) \\
Precipitation time $(\mathrm{s})$ & 40,000 (estimated from ASD data) \\
Particle radius $(\mu \mathrm{m})$ & 25 (for HSWG, RCD tablets), 40 (for RCS tablet) \\
Physiology model & Physiologically fasted human \\
ASF model & Opt LogD SA/V 6.1 \\
\hline
\end{tabular}

${ }^{a}$ Absorption scale factor $(A S F)$ is a multiplier used by GastroPlus to scale the effective surface/volume ratio and other absorption ratedetermining effects of each compartment. The absorption rate coefficient in each compartment is the product of the $\mathrm{P}_{\text {eff }}$ and ASF for that compartment. Opt $\log \mathrm{D}$ Model SA/V 6.1, the current default choice in the program, incorporates pH-partition theory of drug ionization and permeation, and considers the increase in the surface area due to the presence of villi and microvilli in the small intestine

By incorporating in vitro dissolution results into the absorption model to produce biopharmaceutical models, we predicted the in vivo dissolution and absorption profiles of the tablets that were to be tested in Trial 2. The comparison of these profiles among themselves and to those of the solution is presented in Fig. 7. In the solution formulation, galunisertib entered the stomach as a dissolved form and hence there was no initial dissolution phase. However, due to significant solubility decrease upon entry into the duodenum, precipitation from dissolved form into solid drug particles occurred; subsequently, the reduced concentration of galunisertib in the small intestine as the result of precipitation and permeation drove the redissolution of the drug. Such a precipitation-redissolution phenomenon is manifested as a depression that appears within the initial 2-h time window on the dissolution-time profile of solution. The magnitude of this depression is determined by precipitation rate and permeability for the solution formulation. Tablet dissolution profiles clearly demonstrate time-dependent gradual appearance of dissolved drug in the GIT. As compared to solution, there is no obvious depression on the tablet dissolution profiles indicative of the precipitation-redissolution phenomenon. As implied by the absorption-time profiles, complete absorption

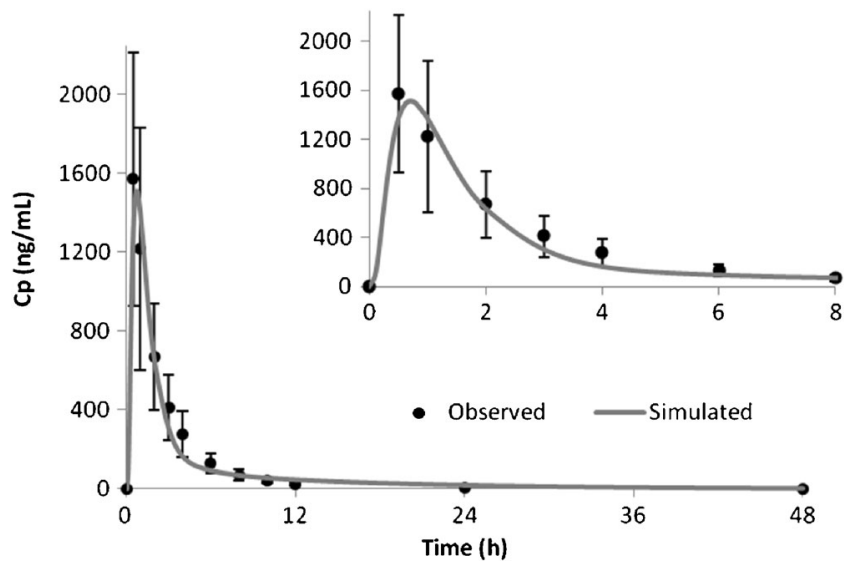

Fig. 6. Simulation of mean plasma concentration-time profiles of galunisertib after oral administration of $150 \mathrm{mg}$ in solution to healthy human subjects in Trial 1. Time is truncated in the inset to better show the fitting of the earlier time points
( $\geq 90 \% F_{a}$ corresponding to $\geq 135 \mathrm{mg}$ of dose absorbed) of galunisertib could be achieved in either solution or tablets. However, it may be absorbed at a significantly lower rate in tablets than in solution. The absorption of galunisertib in solution is near completion (i.e., $90 \% F_{a}$ ) within $4 \mathrm{~h}$; while galunisertib in tablets uses the entire GI transit time $(>16 \mathrm{~h})$ for its absorption. In spite of apparent different dissolution profiles observed in the in vitro dissolution tests and corresponding z's, GastroPlus predicted similar overall in vivo dissolution and absorption profiles for all three tablets (The optimized profiles will be explained later).

By coupling the biopharmaceutical and PK models, we simulated the plasma concentration-time profiles for the tablets and compared to the clinical observations, as shown in Fig. 8. The AUC's of all three tablets estimated by the models are within $90-105 \%$ of the calculations from the observations. The models were able to simulate the performance of RC tablets, but did not work equally well on HSWG tablets. The concentrations of the absorption phase are under-predicted for HSWG tablets, estimated $C_{\max }$ is about $70 \%$ of the observed value, and $T_{\max }$ is significantly delayed. These findings indicate the biopharmaceutical model may under-predict the absorption rate for HSWG tablets. By increasing the gastric $z$ value to $4 \times$ of its initial value, we significantly increased the gastric dissolution rate and apparent absorption rate, as demonstrated by the optimized profiles for HSWG tablets in Fig. 7. Consequently, the optimized biopharmaceutical model greatly improved the simulation of plasma concentration-time for HSWG tablets, as shown in Fig. 8.

\section{DISCUSSION}

A robust and useful absorption model shall be built on valid assumptions on mechanistic sophistication and proper approximations of key inputs. The notion that solubility and permeability are the most important determinants of absorption in the GIT forms the framework of the Biopharmaceutical Classification System (BCS) (37). According to the BCS criteria, Class I compounds have high solubility and permeability which warrant its complete absorption in the gut and generally good prediction by models. For drugs in other 
a

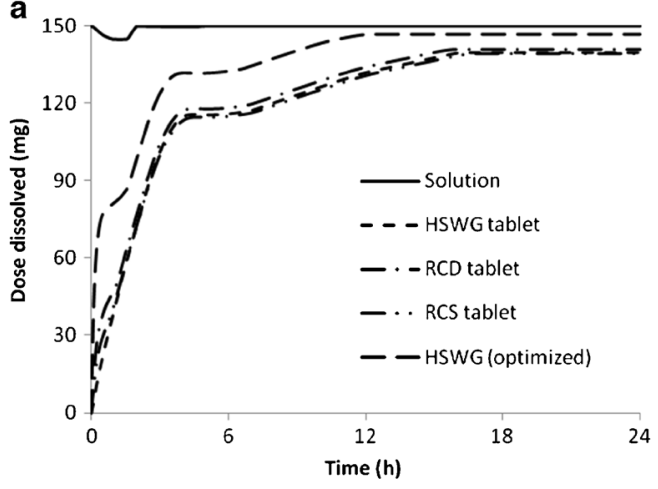

b

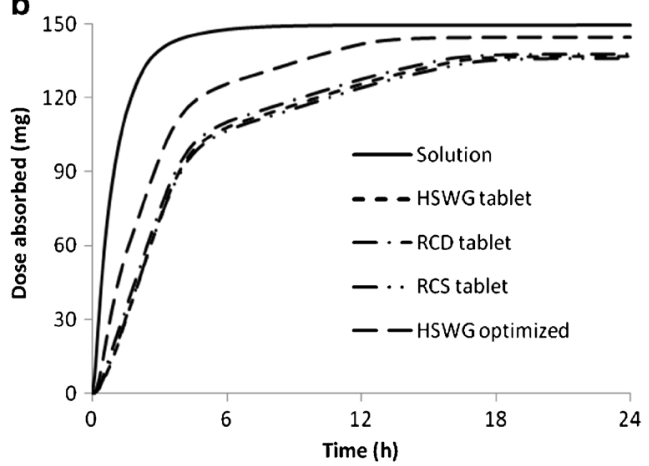

Fig. 7. Simulation of in vivo dissolution (a) and absorption (b) of $150 \mathrm{mg}$ galunisertib in solution or tablets

classes (i.e., II, III, or IV), either solubility or permeability or both can be a limiting factor for the absorption. Therefore, accurate in vivo values of these parameters are critical for models that are able to explain the in vivo behavior already observed and predict the performance under defined circumstances. Solely based on the experimental results (i.e., the lowest equilibrium solubility of $0.05 \mathrm{mg} / \mathrm{mL}$ at $\mathrm{pH} 1-7.4$, not high $\mathrm{P}_{\mathrm{app}}$ on Caco-2 monolayers), galunisertib might be classified as a BCS IV molecule at $150 \mathrm{mg}$ dose. By carefully examining the preclinical in vivo results, we believe this drug is able to permeate the intestinal membrane at a moderately high rate. Such an in silico-in vitro-in vivo approach has been successfully applied to determine effective in vivo permeability for another trial molecule (38). In our experience, such discrepancy of permeability between experimental and in vivo results is fairly common. The in vitro measurements suggest solubility of galunisertib is affected primarily by $\mathrm{pH}$ rather than bile salts. Therefore, the $\mathrm{pH}$-solubility profile in Fig. 2 is believed to provide a generally good estimation for its solubility in vivo. The high sensitivity of solubility to $\mathrm{pH}$ introduces the potential mechanism of supersaturation that could affect the absorption. Hence, precipitation time becomes a third important absorption parameter in need of a good estimation. ASD experiments suggest galunisertib could maintain supersaturation in the intestine for a significantly longer period than 15 min which the default value provided by GastroPlus and often adopted in the absence of experimental values. The significantly slower precipitation greatly minimizes redissolution and thus maximizes drug absorption in the duodenum. According to GastroPlus simulations of the
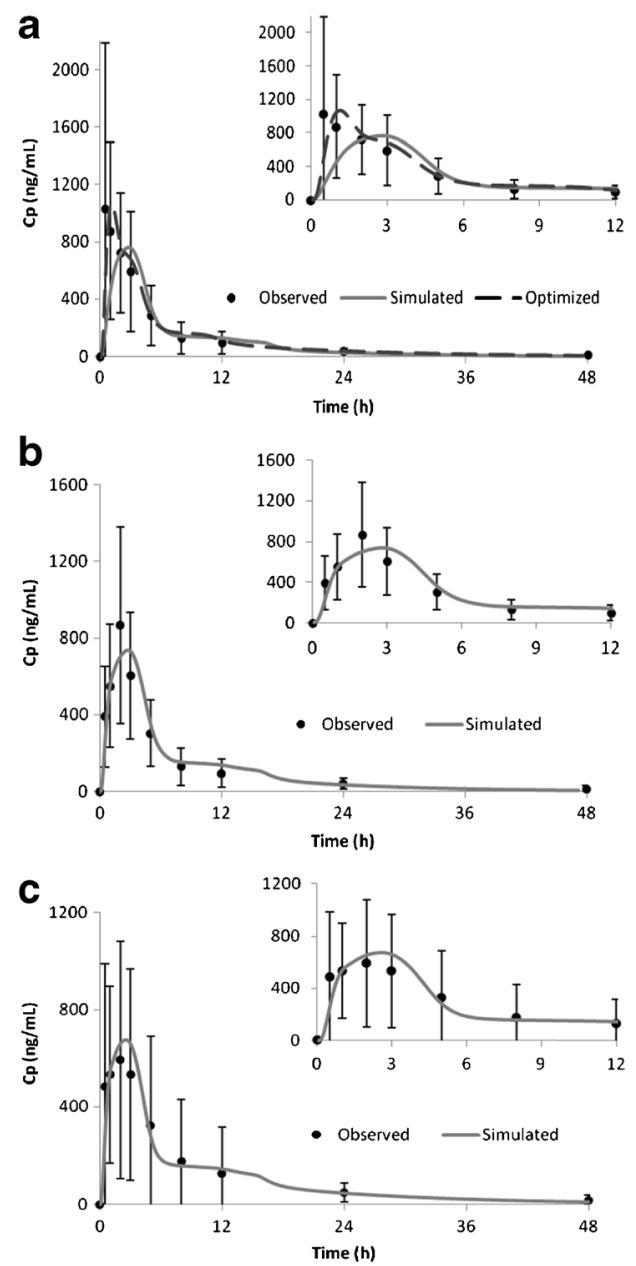

Fig. 8. Simulation of mean plasma concentration-time profile of galunisertib after oral administration to cancer patients in Trial 2. a $3 \times 50 \mathrm{mg}$ HSWG tablet. b $150 \mathrm{mg}$ RCD tablet. c $150 \mathrm{mg}$ RCS tablet. Times are truncated in the insets to better show the fitting of the absorption phases

solution formulation, the dissolved galunisertib could be reduced by $50 \%$ to $75 \mathrm{mg}$ at $1.2 \mathrm{~h}$ with $15 \mathrm{~min}$ of precipitation time, but by less than $4 \%$ to $145 \mathrm{mg}$ at the same time point with $11 \mathrm{~h}$ of precipitation time. The greater amount of dissolved drug in the upper GIT leads to higher $F_{a}$ allocation in the duodenum and jejunum, and possibly earlier and higher concentration peak. After integrating the corrected $\mathrm{P}_{\text {eff }}$ and precipitation time and other absorption parameters into models, we estimated complete absorption ( $>90 \%$ ) for galunisertib of $150 \mathrm{mg}$ in vivo, and were able to overturn the initial BCS IV classification only based on discrete experimental data.

Effective integration of dissolution tests and absorption modeling could create a powerful tool to assess the effects of biopharmaceutical properties and formulation effects on the release of the drug from its products, subsequent dissolution, and absorption in the GIT. Such integrative results are presented in Figs. 7 and 8. Although in the in vitro multimedia tests the initial dissolution rates of galunisertib in HSWG tablets were different than in RC tablets (i.e., slower in $\mathrm{pH} 2$ but faster in other media), and accordingly different $z$ 's were calculated by GastroPlus, simulations did not predict considerable difference in in vivo dissolution and absorption in these formulations. When comparing the performance of 
tablets and solution, simulations suggested release and dissolution of the drug from solid formulations may limit the absorption of galunisertib. The limitation is primarily on the rate of absorption indicated by $C_{\max }$ and $T_{\max }$, rather than the extent of absorption (i.e., $F_{a}$ ). $F_{a}$ of any of these tablets could have reached $90 \%$, the borderline "complete absorption" defined in BCS criteria. Slow dissolution in the stomach could also reduce the benefit of supersaturation for the absorption. Under such a circumstance, not all the dose could have been dissolved and maintained in supersaturation for absorption when entering the duodenum. Some solid drug particles would have to be dissolved at a much slower rate in the duodenum prior to absorption. Therefore, faster dissolution in the stomach is critical for this basic drug to achieve higher and earlier $C_{\max }$. We could effectively improve the fitting for HSWG tablets by increasing the gastric z-factor, the parameter for the dissolution in the stomach. Using parameter sensitivity analysis, we found this $z$ must be increased to $4 \times$ or higher of its initial value for the good fit. Although faster gastric dissolution of galunisertib in HSWG tablets apparently is not supported by USP dissolution test in $0.01 \mathrm{~N} \mathrm{HCl}$ due to complication by in vitro disintegration, the dynamic dissolution tests in ASD demonstrated more galunisertib molecules could be dissolved in the stomach and duodenum. In contrast to the results of USP dissolution test, we did not observe slower disintegration of HSWG tablets and the consequent impact on initial apparent dissolution rate in ASD experiments. The different performances in both tests that might be attributed to the dynamic conditions presented some difficulty in choosing a more predictive in vitro test. The exposures of dissolved drug in ASD fluid have been successfully used to rank order systemic AUC and $C_{\max }$ for several carbamazepine products (39). Therefore, adopting the same method, we could predict the rank order of in vivo AUC and $C_{\max }$ for these tablets as $\mathrm{HSWG}>\mathrm{RCS}=\mathrm{RCD}$, which was consistent with the clinical observation. Parameter sensitivity analysis in GastroPlus demonstrated faster gastric dissolution could lead to higher exposure of dissolved drug in the upper small intestine and $C_{\max }$ in systemic circulation. Such outcomes are implicitly suggested in Fig. 7b where optimized HSWG dissolution in the stomach results in higher amount of dose absorbed during the early transit. Unfortunately, we have yet been able to quantitatively correlate difference of exposures in ASD with observed systemic $C_{\max }$ 's in this case. It is speculated that different dynamic setup to simulate GI physiology for absorption between in vitro ASD tests and GastroPlus program, such as lack of drug permeation in ASD and fluid transfer kinetics, could add complexity to such a direct correlation. Given the great interest in biorelevant dynamic dissolution tests that are believed to be a better approximation of in vivo dissolution, identifying a robust method of integrating ASD test results into absorption modeling may be a worthwhile endeavor.

As discussed previously, z-factor dissolution model allows us to incorporate USP dissolution test results into modeling to accurately predict comparable AUC's for different galunisertib products, but apparently it did not perform equally well in prediction of $C_{\text {max }}$. In practice, $z$ is calculated primarily by fitting time-dissolved concentration profile with Eq. 4. Hence, the accuracy of the calculation depends on the accuracy of other input parameter values in the equation, including the dissolved mass measured in the process of dissolution, and solubility at the specific $\mathrm{pH}$. Due to its presumed high solubility at very low $\mathrm{pH}$, the saturated drug solution elevated the $\mathrm{pH}$ of $0.1 \mathrm{~N} \mathrm{HCl}$ to 2.7 during the solubility measurement. The inability of obtaining accurate solubility at $\mathrm{pH} 2$ or below (the default gastric $\mathrm{pH}$ for fasted human is 1.3 in GastroPlus physiological model) inevitably affected the derivation of $z$ 's from the fitting. The tolerance of biopharmaceutical modeling to uncertainty of $z$ depends on the sensitivity of specific outputs to this input. In this case, $F_{a}$ and AUC are only slightly changed by optimization; however, $C_{\max }$ and $T_{\max }$ are affected greatly with $45 \%$ increase in $C_{\max }$ and a half-cut $T_{\max }$. On the one hand, this finding indicates $z$ can be an important parameter to consider in optimization of the fitting or assessing variability; on the other hand, the uncertainty in $z$ could impact the predictability of a biopharmaceutical model. The general probability of good in vitro-in vivo correlation of $z$ is not yet clear. In this case, from the USP dissolution test, $z$ seems to be sensitive to $\mathrm{pH}$. Considerable variability of gastric $\mathrm{pH}$ in fasted human can be introduced by physiological variations $(40,41)$, pathological conditions (42), pharmacological effects (43), etc. In our opinion, in the absence of mechanistic understanding and a theoretical relationship between $\mathrm{pH}$ and $z$ (considering the Henderson-Hasselbalch equation for the $\mathrm{pH}$-solubility effect as a classic example), extrapolation or interpolation becomes difficult and less reliable. In this case, we used discreet zfactors for gastric and intestinal dissolution as a resolution. Such simplification may cost the accuracy of in vivo $z$ estimation. If the inaccuracy is too high to ensure a good approximation of the key input value, quality of simulations is likely affected. By this brief critical review of $\mathrm{z}$-dissolution model based on our experience from this case, we hope to stimulate more interest and encourage more effort in improving the tools for biopharmaceutical modeling.

\section{CONCLUSION}

By carefully designing in vitro experiments, analyzing experimental and preclinical data, we constructed integrative physiologically based biopharmaceutical models to evaluate the in vivo performance of typical clinical products for a basic drug candidate. High permeability, sufficient in vivo solubility, and propensity to supersaturation on the absorption site enable the drug to achieve complete clinical absorption at a clinically relevant dose. The rate of absorption may be more susceptible to the formulation effect. The in vitro dynamic dissolution test ASD and z-factor dissolution model show some promise in integrating in vitro product performance assessment with modeling. More investment is in need to explore and advance such in vitro-in silico approaches to better understand and predict in vivo performance of drug products.

\section{REFERENCES}

1. Martinez MN, Amidon GL. A mechanistic approach to understanding the factors affecting drug absorption: a review of fundamentals. J Clin Pharmacol. 2002;42(6):620-43.

2. Lennernäs H. Human intestinal permeability. J Pharm Sci. 1998;87(4):403-10.

3. Karlsson J, Ungell A-L, Grasjo J, Arturrson P. Paracellular drug transport across intestinal epithelia: influence of charge and induced water efflux. Eur J Pharm Sci. 1999;9(1):47-56. 
4. Ayrton A, Morgan P. Role of transport proteins in drug absorption, distribution and excretion. Xenobiotica. 2001;31(8-9):469-97.

5. Hou T, Wang J, Zhang W, Wang W, Xu X. Recent advances in computational prediction of drug absorption and permeability in drug discovery. Curr Med Chem. 2006;13(22):2653-67.

6. Sjögren E, Abrahamsson B, Augustijns P, Becker D, Bolger MB, Brewster $\mathrm{M}$, et al. In vivo methods for drug absorption-comparative physiologies, model selection, correlations with in vitro methods (IVIVC), and applications for formulation/API/excipient characterization including food effects. Eur J Pharm Sci. 2014;57:99-151.

7. Noyes AA, Whitney WR. The rate of solution of solid substances in their own solutions. J Am Chem Soc. 1897;19:930-4.

8. Johnson KC, Swindell AC. Guidance in the setting of drug particle size specifications to minimize variability in absorption. Pharm Res. 1996;13(12):1795-8.

9. He X. Integration of physical, chemical, mechanical and biopharmaceutical properties in solid oral dosage form development. In: Qiu Y, Chen Y, Zhang G, Liu L, Porter W, editors. Developing solid oral dosage forms: pharmaceutical theory \& practice. 1st ed. Oxford: Elsevier Inc; 2008. p. 407-41.

10. Mishra M, Muthuprasanna P, Prabha KS, Rani PS, Babu IAS, Chandiran $\mathrm{S}$, et al. Basics and potential applications of surfactants—a review. Int J Pharm Tech Res. 2009;1(4):1354-65.

11. Dressman JB, Amidon GL, Reppas C, Shah VP. Dissolution testing as a prognostic tool for oral drug absorption: immediate release dosage forms. Pharm Res. 1998;15(1):11-22.

12. Emami J. In vitro-in vivo correlations: from theory to applications. J Pharm Sci. 2006;9(2):169-89.

13. Klein $\mathrm{S}$. The use of biorelevant dissolution media to forecast the in vivo performance of a drug. AAPS J. 2010;12(3):397-406.

14. Kostewicz ES, Abrahamsson B, Brewster M, Brouwers J, Butler $\mathrm{J}$, Carlert $\mathrm{S}$, et al. In vitro models for the prediction of in vivo performance of oral dosage forms. Eur J Pharm Sci. 2014;57:34266.

15. McAllister M. Dynamic dissolution: a step closer to predictive dissolution testing? Mol Pharm. 2010;7(5):1374-87.

16. Castela-Papin N, Cai S, Vatier J, Keller F, Souleau CH, Farinotti R. Drug interactions with diosmectite: a study using the artificial stomach-duodenum model. Int $\mathrm{J}$ Pharm. 1999;182(1):111-9.

17. Vatier J, Celice-Pingaud C, Farinotti R. A computerized artificial stomach model to assess sodium alginate-induced $\mathrm{pH}$ gradient. Int J Pharm. 1998;163(1-2):225-9.

18. Bhattachar SN, Perkins EJ, Tan JS, Burns LJ. Effect of gastric $\mathrm{pH}$ on the pharmacokinetics of a BCS class II compound in dogs: utilization of an artificial stomach and duodenum dissolution model and GastroPlus, simulations to predict absorption. J Pharm Sci. 2011;100(11):4756-65.

19. Takano R, Takata N, Saito R, Furumoto K, Higo S, Hayashi Y, et al. Quantitative analysis of the effect of supersaturation on in vivo drug absorption. Mol Pharm. 2010;7(5):1431-40.

20. Jiang W, Kim S, Zhang X, Lionberger RA, Davit BM, Conner DP, et al. The role of predictive biopharmaceutical modeling and simulation in drug development and regulatory evaluation. Int $\mathbf{J}$ Pharm. 2011;418(2):151-60.

21. Mathias NR, Crison J. The use of modeling tools to drive efficient oral product design. AAPS J. 2012;14(3):591-600.

22. Kostewicz ES, Aarons L, Bergstrand M, Bolger MB, Galetin A, Hatley $\mathrm{O}$, et al. PBPK models for the prediction of in vivo performance of oral dosage forms. Eur J Pharm Sci. 2014;57C:300-21.

23. Zhang $\mathrm{X}$, Lionberger RA, Davit BM, Yu LX. Utility of physiologically based absorption modeling in implementing quality by design in drug development. AAPS J. 2011;13(1):5971.

24. Yu L, Zhang X, Lionberger R. Modeling and mechanistic approaches for oral absorption: quality by design in action. Ther Deliv. 2012;3(2):147-50.

25. Balimane PV, Han YH, Chong S. Current industrial practices of assessing permeability and P-glycoprotein interaction. AAPS J. 2006;8(1):E1-13.

26. Van Breeman $\mathrm{RB}, \mathrm{Li} \mathrm{Y}$. Caco-2 cell permeability assays to measure drug absorption. Expert Opin Drug Metab Toxicol. 2005;1(2):175-85.

27. Agoram B, Woltosz WS, Bolger MB. Predicting the impact of physiological and biochemical processes on oral drug bioavailability. Adv Drug Deliv Rev. 2001;50 Suppl 1:S41-67.

28. Poulin P, Theil FP. A priori prediction of tissue:plasma partition coefficients of drugs to facilitate the use of physiologically-bed pharmacokinetic models in drug discovery. J Pharm Sci. 2000;89(1):16-35.

29. Rodgers T, Leahy D, Rowland M. Physiologically-based pharmacokinetic modeling 1: predicting the tissue distribution of moderate-to-strong bases. J Pharm Sci. 2005;94(6):1259-76.

30. Berezhkovskiy LM. Volume of distribution at steady state for a linear pharmacokinetic system with peripheral elimination. J Pharm Sci. 2004;93(6):1628-40.

31. Hintz RJ, Johnson KC. The effect of particle size distribution on dissolution rate and oral absorption. Int J Pharm. 1989;51:9-17.

32. Takano R, Sugano K, Higashida A, Hayashi Y, Machida M, Aso $\mathrm{Y}$, et al. Oral absorption of poorly water-soluble drugs: computer simulation of fraction absorbed in humans from a miniscale dissolution test. Pharm Res. 2006;23(6):1144-56.

33. Ding X, Rose JP, van Gelder J. Clinical product developability assessment with maximum absorbable dose. Int $\mathrm{J}$ Pharm. 2012;427:260-9.

34. Neuhoff S, Ungell A-L, Zamora I, Artusson P. PH-Dependent bidirectional transport of weakly basic drugs across Caco-2 monolayers: implications for drug-drug interactions. Pharm Res. 2003;20(8):1141-8.

35. Neuhoff S, Ungell A-L, Zamora I, Artusson P. PH-Dependent passive and active transport of acidic drugs across Caco- 2 cell monolayers. Eur J Pharm Sci. 2005;25(2-3):211-20.

36. Polster CS, Wu S-J, Gueorguieva I, Sperry DC. Mechanism for enhanced absorption of a solid dispersion formulation of LY2300559 using the artificial stomach duodenum model. Mol Pharmaceutics. 2015;12(4):1131-40.

37. Amidon GL, Lennernas H, Shah VP, Crison JR. A theoretical basis for a biopharmaceutic drug classification: the correlation of in vitro drug product dissolution and in vivo bioavailability. Pharm Res. 1995;12(3):413-20.

38. Ding X, He M, Kulkarni R, Patel N, Zhang X. Investigation of clinical pharmacokinetic variability of an opioid antagonist through physiologically based absorption modeling. J Pharm Sci. 2013;102(8):2859-74.

39. Carino SR, Sperry DC, Hawley M. Relative bioavailability estimation of carbamazepine crystal forms using an artificial stomach-duodenum model. J Pharm Sci. 2006;95(1):116-25.

40. Mudie DM, Amidon GL, Amidon GE. Physiological parameters for oral delivery and in vitro testing. Mol Pharmaceutics. 2010;7(5):1388-405.

41. Washington $\mathrm{N}$, Washington $\mathrm{C}$, Wilson C. Physiological pharmaceutics barriers to drug absorption. 2nd ed. London: Taylor \& Francis; 2002. p. 75-108.

42. Huang JQ, Hunt RH. pH, healing rate, and symptom relief in patients with GERD Yale. J Biol Med. 1999;72(2-3):181-94.

43. Zhang L, Wu F, Lee SC, Zhao H, Zhang L. pH-dependent drugdrug interactions for weak base drugs: potential implications for new drug development. Clin Pharmacol Ther. 2014;96(2):266-77. 